\title{
Quantum Neuromorphic Platform for Quantum State Preparation
}

\author{
Sanjib Ghosh $\odot,{ }^{1,2, *}$ Tomasz Paterek $\odot,{ }^{1,2,3}$ and Timothy C. H. Liew ${ }^{1,2, \dagger}$ \\ ${ }^{1}$ School of Physical and Mathematical Sciences, Nanyang Technological University, 637371 Singapore \\ ${ }^{2}$ MajuLab, International Joint Research Unit UMI 3654, CNRS, Université Côte d'Azur, Sorbonne Université, \\ National University of Singapore, Nanyang Technological University, Singapore \\ ${ }^{3}$ Institute of Theoretical Physics and Astrophysics, Faculty of Mathematics, Physics and Informatics, \\ University of Gdańsk, 80-308 Gdańsk, Poland
}

(Received 25 July 2019; published 27 December 2019)

\begin{abstract}
We develop a scheme of quantum reservoir state preparation, based on a quantum neural network framework, which takes classical optical excitation as input and provides desired quantum states as output. We theoretically demonstrate the broad potential of our scheme by explicitly preparing a range of intriguing quantum states, including single-photon states, Schrödinger's cat states, and two-mode entangled states. This scheme can be used as a compact quantum state preparation device for emerging quantum technologies.
\end{abstract}

DOI: 10.1103/PhysRevLett.123.260404

In quantum physics, the description of the physical world is given in terms of quantum states that allow nonclassical correlations between different objects and measurement outcomes. Taking advantage of these quantum properties, quantum technologies, e.g., quantum long distance communication [1], information processing [2], computation [3], and more recently quantum internet [4], are rising. Whether operating with continuous or discrete variables, quantum technologies require preparing suitable quantum states, e.g., Schrödinger's cat states, single-photon states, and entangled states to name a few.

Even though typically restricted to the classical domain, neural networks have become powerful machines for the identification of correlations in input data. At the same time, their massively parallel architectures with many interconnections mapping to different outputs are natural for generating outputs that are correlated. Neural networks have formed efficient representations of quantum states (e.g., with restricted Boltzmann machines [5-8]) and can reconstruct highly correlated patterns given an imperfect input (i.e., associative memories, which were also generalized to the quantum regime [9]).

While most forms of neural networks are used as software for preexisting hardware, reservoir computing, which is a recurrent neural network, is particularly suitable for neuromorphic implementation over a wide range of physical systems [10-13]. In reservoir computing, an

Published by the American Physical Society under the terms of the Creative Commons Attribution 4.0 International license. Further distribution of this work must maintain attribution to the author(s) and the published article's title, journal citation, and DOI. interconnected network, known as the reservoir, is used for mapping the input signal into its large nonlinear feature space, where virtually any output function can be approximated as a linear cut. This large network (reservoir) is taken as fixed and random, which is the main reason behind its hardware success. Reservoir computing follows the prescription of artificial neural networks that learn from training to perform various tasks. However, the training process in reservoir computing takes place only at the output level. Consequently, training in reservoir computing is a linear process and is easier to perform than in other forms of recurrent neural networks $[14,15]$.

While the applications of reservoir computing largely focused on classical tasks (even with quantum reservoirs [16]), the idea was recently brought fully into the quantum world in the form of quantum reservoir processing [17]. It was shown as an efficient platform for quantum entanglement recognizing tasks and for performing complex quantum measurements (e.g., entropy, purity, and negativity). Although performing these tasks involves processing the quantum nature of the input states, the output is still classical data obtained through measurements on the reservoir. Here we invert the paradigm of quantum reservoir processing, replacing a quantum input with a classical one and classical output with a quantum one, to arrive at a neuromorphic platform for quantum reservoir state preparation (QRSP), as illustrated in Fig. 1. In this scheme, a classical source excites a quantum reservoir where the signal evolves and disperses into the large Hilbert space of the quantum reservoir. Because of the quantum nonlinear nature of the reservoir, the emission from the reservoir constitutes a quantum field. To obtain the final output quantum state, the emitted field is processed with a suitable linear optical setup (corresponding to matrix multiplication 


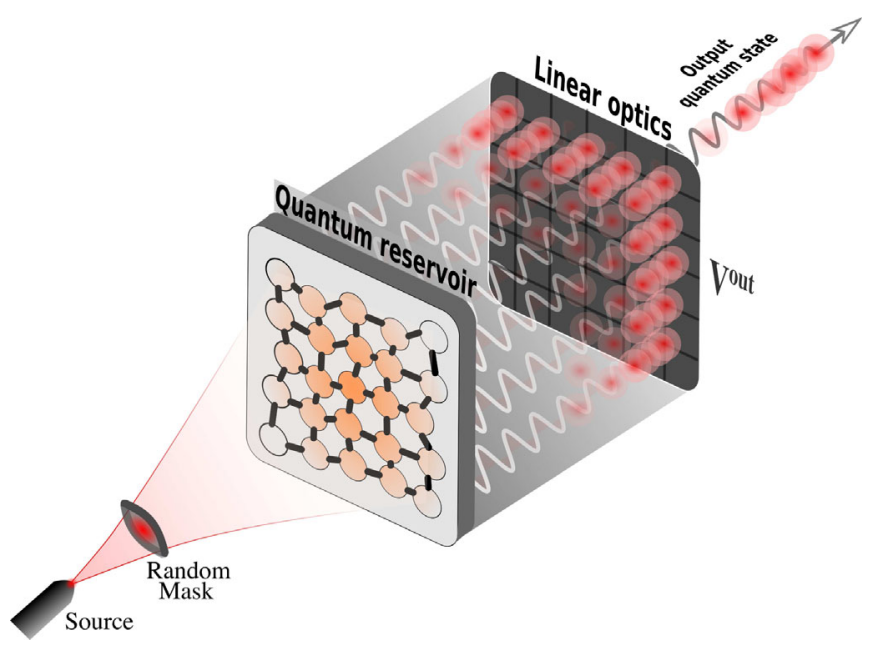

FIG. 1. A scheme of quantum reservoir state preparation. We considered a set of nonlinear bosonic sites arranged in a 2D disordered square lattice (with coupling between nearest neighbors) as the reservoir. This is excited with a classical randomly distributed optical field (generated by a random mask). The emission from the reservoir is then taken through a linear optical setup represented by the output weight matrix $\mathbf{V}^{\text {out }}$. The final output state is given by a linear combination of the quantum fields emitted from the reservoir.

of a vector of outputs) where the training takes place. This platform can be used for preparation of different types of quantum states.

Result. - In our consideration, the quantum reservoir is a nonlinear 2D square lattice with the size $N$ (total number of lattice points) that is optically excited with a randomly distributed classical field. This reservoir is represented by the Hamiltonian:

$$
\begin{aligned}
\hat{H}= & \sum_{i} E_{i} \hat{a}_{i}^{\dagger} \hat{a}_{i}+\sum_{\langle i j\rangle} J_{i j}\left(\hat{a}_{i}^{\dagger} \hat{a}_{j}+\hat{a}_{j}^{\dagger} \hat{a}_{i}\right) \\
& +\sum_{i} \alpha_{i} \hat{a}_{i}^{\dagger} \hat{a}_{i}^{\dagger} \hat{a}_{i} \hat{a}_{i}+\sum_{i}\left(P_{i} \hat{a}_{i}^{\dagger}+P_{i}^{*} \hat{a}_{i}\right) .
\end{aligned}
$$

Here, $J_{i j}, E_{i}, \alpha_{i}$, and $P_{i}$ are the randomly distributed and site-dependent nearest-neighbor hopping amplitudes, onsite energies, nonlinearity strengths, and classical optical excitations, respectively. We consider that the excitation field is coherent, which could be arranged by passing the output from a single laser source through a spatial light modulator (or amplitude mask). For the hopping amplitudes, we consider that $J_{i j}$ are random parameters uniformly distributed in the interval $[-1,+1]$ and then normalized such that the spectral radius (largest magnitude eigenvalue) of the hopping part of the Hamiltonian is $\tilde{J}$. The spectral radius sets the energy scale for the Hamiltonian. The quantum master equation for the density matrix of the reservoir $\rho$ is given by [18-20]

$$
\dot{\rho}=-i[\hat{H}, \rho]+\frac{1}{2} \sum_{j}\left(2 \hat{a}_{j} \rho \hat{a}_{j}^{\dagger}-\hat{a}_{j}^{\dagger} \hat{a}_{j} \rho-\rho \hat{a}_{j}^{\dagger} \hat{a}_{j}\right),
$$

where we expressed all energies and times in units of the linewidth $\gamma$ (assumed the same for all reservoir sites) and $\hbar / \gamma$, respectively. The Lindblad dissipation term [last term in Eq. (2)] represents coupling to a Markovian environment with continuous energy spectrum, which often appears for systems with optical losses. The quantum fields emitted (in a transmission geometry as shown in Fig. 1) by each reservoir site are given by $\hat{a}_{j}[18]$. We assume that the standard toolbox of linear optical elements is available [2] for combining the transmitted fields to generate $M$ final output modes of the form

$$
\hat{A}_{i}=\sum_{j} v_{i j} \hat{a}_{j}
$$

where $v_{i j}$ are complex coefficients. The output quantum modes $\hat{A}_{i}$ must satisfy the commutation relations $\left[\hat{A}_{i}, \hat{A}_{j}^{\dagger}\right]=$ $\delta_{i j}$ imposing the conditions

$$
\sum_{k} v_{i k} v_{j k}^{*}=\delta_{i j} .
$$

These coefficients form a vector space with the unit vectors $\mathbf{v}_{i}=\left(v_{i 1}, v_{i 2}, \ldots, v_{i N}\right)$ and satisfy orthogonality:

$$
\mathbf{v}_{i} \cdot \mathbf{v}_{j}^{*}=\delta_{i j} .
$$

The number of such unit vectors $M$ can be less than or equal to $N$, as one can construct at most as many independent output modes as the number of reservoir sites. In the following we will consider two situations, one with $M=1$ and the other with $M=2$. The output weights $\mathbf{V}^{\text {out }}$ with the elements $v_{i j}$ and with the structure of an $N \times M$ matrix would be obtained by training. For training we numerically optimize cost functions that are extremum when the prepared states become the same as the desired states. For optimization we use the Nelder-Mead simplex algorithm [21]. More details on the numerical methods are given in the Supplementary Material (SM) [22].

Single-photon states.-Single photons are important resources for various quantum technologies. Unless postprocessing is used, their generation typically requires a system with strong photon-photon interaction, such that their sensitivity to occupation number and antibunching can be engineered. However, in practice, achieving strong photon-photon interaction is a challenging task. Here, we show that a QRSP system can induce strong antibunching even in the weakly interacting regime (where the photonphoton interaction is much smaller than the linewidth, i.e., $\left.\alpha_{i}<\gamma\right)$. We consider single-mode antibunching by assigning $M=1$. The corresponding output occupation number $\langle\hat{n}\rangle$ and the second order correlation function $g_{2}$ are defined by 

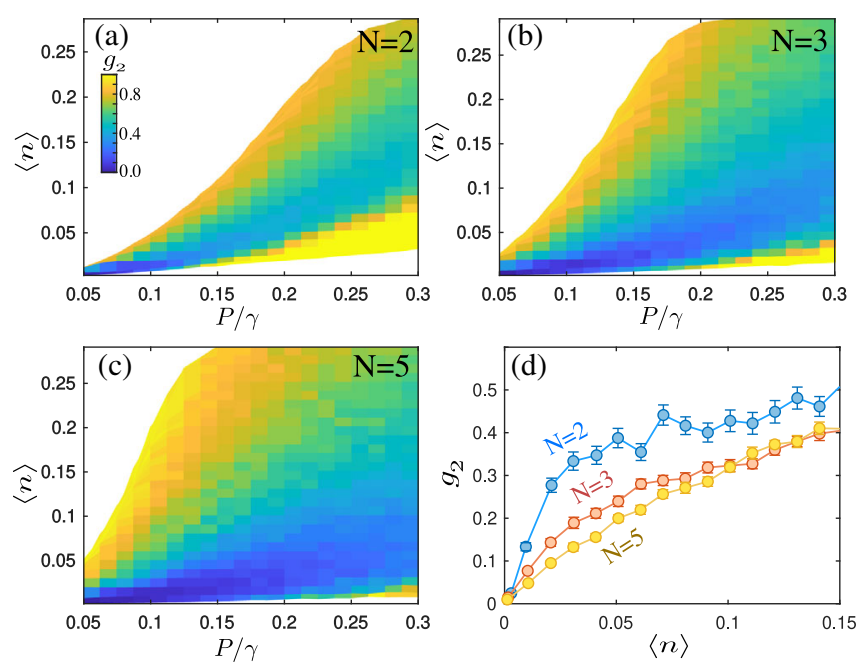

FIG. 2. Color plots of $g_{2}$ averaged over many reservoir configurations as a function of occupation number $\langle\hat{n}\rangle$ and the coherent excitation strength $P\left(P_{i}\right.$ are taken random from the interval $[0,1] \times P)$ for reservoir sizes 2,3 , and 5 shown in (a)-(c), respectively. The dark blue areas indicate the strong antibunching regime (low $g_{2}$ ) where single-photon states form. In (d), we show $g_{2}$ as a function of occupation number with optimum values of $P$ for different reservoir size. Note that an ideal single-photon source requires a low $g_{2}$ with a large $\langle\hat{n}\rangle$. Here, we consider uniform characteristics of the lattice sites, namely $\alpha_{i}=\alpha$ and $E_{i}=E$, and we have chosen $\alpha / \gamma=0.15$ (this value is close to the value extracted from the experimental measurements in Ref. [23]), $E=0$, and $\tilde{J} / \gamma=0.5$.

$$
\langle\hat{n}\rangle=\left\langle\hat{A}_{1}^{\dagger} \hat{A}_{1}\right\rangle, \quad g_{2}=\frac{\left\langle\hat{A}_{1}^{\dagger} \hat{A}_{1}^{\dagger} \hat{A}_{1} \hat{A}_{1}\right\rangle}{\langle n\rangle^{2}} .
$$

We optimize $\mathbf{V}^{\text {out }}$ such that $g_{2}$ (corresponding to the cost function) is minimum. In Fig. 2, we show $g_{2}$ in the steady state as a function of the output occupation number $\langle\hat{n}\rangle$ and the uniform excitation strength $P$. We find that, in general, the antibunching effect becomes weaker with increasing occupation number. However, with increasing size of the reservoir one can reach smaller $g_{2}$ for a fixed $\langle\hat{n}\rangle$.

We find that QRSP applies a generalized form of unconventional blockade [19] for inducing the antibunching effect. In the unconventional blockade, strong antibunching forms due to the destructive interference of pathways leading to two-photon occupation [in particular, the pathways $|10\rangle \rightarrow|20\rangle$ and $|10\rangle \rightarrow|01\rangle \rightarrow(|11\rangle \leftrightarrow$ $|02\rangle) \rightarrow|20\rangle$, where $\left|n_{1} n_{2}\right\rangle$ is the Fock state with $n_{1}$ particles in the first site and $n_{2}$ particles in the second; here the double arrow induces an extra phase in the second pathway leading to the destructive interference] [20]. In QRSP, the suppression of multiphoton emissions is generalized to higher orders.

To explicitly demonstrate, we consider reservoirs in different configurations [Figs. 3(a)-3(e)] and show the corresponding photon number distribution $\mathcal{P}_{n}=$ $\left\langle\psi_{n}|\rho| \psi_{n}\right\rangle$ (where Fock state $\left|\psi_{n}\right\rangle=\left(\hat{A}_{1}^{\dagger n} / \sqrt{n !}\right) \mid$ vac $\rangle$ and $|v a c\rangle$ is the vacuum state). We find among the two-site reservoirs, the configuration [Fig. 3(c)] with unequal excitation and complex output weights shows the strongest antibunching. The unconventional blockade [Fig. 3(a), this is the original configuration [19] ] induces reduction in the two-photon emission probability $\mathcal{P}_{2}[24,25]$. However, the configuration shown in Fig. 3(c) shows reductions in higher order photon emission probabilities $\mathcal{P}_{n>2}$ with respect to the same for other configurations. Because of this suppression of higher order photon emission, the antibunching effect becomes stronger. A similar conclusion had already been reached in Ref. [26]; however, in considering the system as a reservoir neural network it is instructive to consider further increasing the system size. Indeed, we find that larger reservoirs [e.g., Fig. 3(e)] result in overall reduction of multiphoton occupation (left-hand panel in Fig. 3) and further enhancement of antibunching [Fig. 2(d)]. While the state-of-the-art experiment [23] shows $g_{2} \approx 0.95$, our scheme predicts $g_{2} \approx 0.1$ for a similar nonlinearity strength.

Schrödinger's cat states.-Here we show how QRSP can be used to prepare Schrödinger's cat states, $\mid$ Cat $\rangle \equiv$ $(|+\zeta\rangle+|-\zeta\rangle) / \sqrt{2}$, which are an intriguing superposition of two classically distinct coherent states, $| \pm \zeta\rangle=$ $\exp \left(-|\zeta|^{2} / 2 \pm \zeta \hat{A}_{1}^{\dagger}\right)|\mathrm{vac}\rangle$. For preparing these states with QRSP, we consider a single output mode with $M=1$.

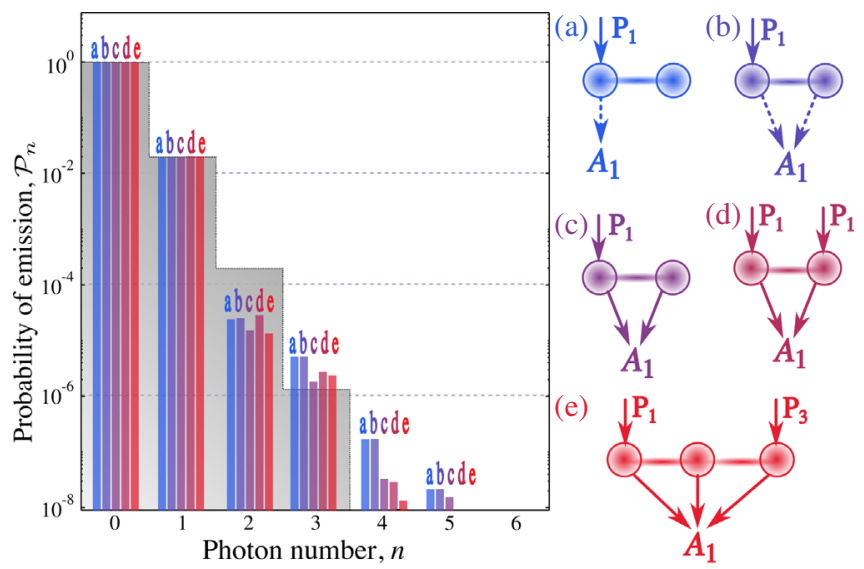

FIG. 3. Photon number distributions for different configurations. On the left, we plot the probability $\mathcal{P}_{n}$ of $n$-photon emission with a fixed occupation number $\langle\hat{n}\rangle=0.02$ for the considered five configurations (a)-(e) shown on the right (the background distribution in gray shows $\mathcal{P}_{n}$ for coherent light with the same occupation number). On the right, we show the following two-site configurations: (a) one site is excited, and emission from the same site is taken as the output; (b) one site is excited, and all emissions with real weights are taken for output; (c) one site is excited, and all emissions with complex weights are taken for output; (d) all sites are excited with the same strength, and all emissions with complex weights are taken for output. Panel (e) shows a three-site reservoir with unequal excitation strengths, and all emissions with complex weights are taken for output. 

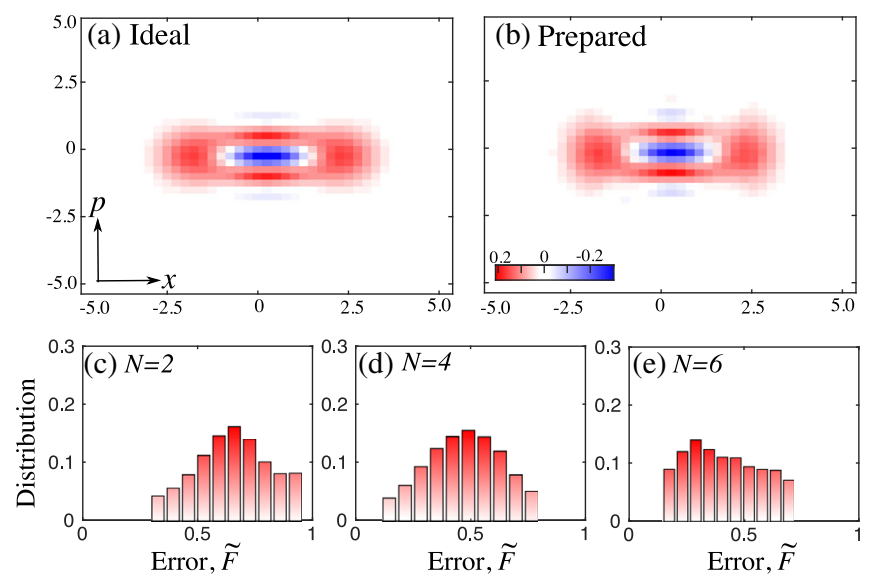

FIG. 4. An example of an ideal cat state (a) compared with the prepared one (b). Here we used a quantum reservoir with only 5 sites. The error of the prepared state is 0.085 . In (c)-(e), we show histograms of errors of the prepared cat states with different realizations of the reservoir for increasing reservoir sizes. As the reservoir size $N$ increases, the chance of preparing low error states increases. For cat state preparation, we consider the parameters $\zeta=1.4$, propagation time $\tau=1.3 \times 10^{-2} \hbar / \gamma$, $\tilde{J} / \gamma=5 \times 10^{2}$, and the random parameters $P_{i} / \gamma, \alpha_{i} / \gamma$, and $E_{i} / \gamma$ are uniformly distributed in $[-1,1] \times 10^{3},[0.5,1.5] \times 10^{3}$ and $[-0.5,0.5] \times 10^{3}$, respectively.

For our demonstration, we consider $\zeta=1.4$ for numerical calculation, which sets the expectation value of the occupation number $\left\langle\hat{A}_{1}^{\dagger} \hat{A}_{1}\right\rangle \simeq \zeta^{2}$. We excite the reservoir with continuous excitation for a time interval $\tau$, and then use the state of the reservoir sites for preparing the output cat state. The Wigner function representing the cat state is shown in Fig. 4(a). In Fig. 4(b), we show the Wigner function of a state prepared with QRSP (see SM for details [22]). As a measure of inaccuracy in the prepared state we define an error:

$$
\tilde{F}=\sqrt{\frac{\sum_{i j}\left[W\left(x_{i}, p_{j}\right)-W^{\prime}\left(x_{i}, p_{j}\right)\right]^{2}}{\sum_{i j}\left[W\left(x_{i}, p_{j}\right)+W^{\prime}\left(x_{i}, p_{j}\right)\right]^{2}}},
$$

where $W$ and $W^{\prime}$ are the Wigner functions of the ideal and prepared state, respectively. For training, we consider $\tilde{F}$ as the cost function and minimize it with an appropriate choice of $\mathbf{V}^{\text {out }}$. Equivalently, one can optimize the fidelity of the output state for training (see SM [22]). The errors are shown in Figs. 4(c)-4(e) considering different realizations of the reservoir, i.e., $J_{i j}$. We find that larger reservoirs allow smaller errors, while there is always some spread in the errors depending on the chosen $J_{i j}$. Thus additional optimization over the reservoir can give benefits in terms of the system performance as well.

Entangled states.-We prepare two-mode entangled states with the same scheme as followed for preparing the Schrödinger's cat states, but here with $M=2$. In the two-mode output subspace, we calculate the negativity $\left[\mathrm{Ne}\left(\rho_{\text {out }}\right)\right]$ from the corresponding output density matrix $\rho_{\text {out }}$. The negativity $\operatorname{Ne}\left(\rho_{\text {out }}\right)$ is defined as the sum of the negative eigenvalues of $T_{1}\left[\rho_{\text {out }}\right]$, where $T_{1}[\cdots]$ represents the partial transpose with respect to the output mode $\hat{A}_{1}$. We then train the output weights $\mathbf{V}^{\text {out }}$ such that $\operatorname{Ne}\left(\rho_{\text {out }}\right)$ is the maximum possible. Given the $\mathbf{V}^{\text {out }}=\left(\mathbf{v}_{1}, \mathbf{v}_{2}\right)$, we form the output modes:

$$
\begin{aligned}
& \hat{A}_{1}=\mathbf{v}_{1} \cdot \hat{\mathbf{a}}, \\
& \hat{A}_{2}=\mathbf{v}_{2} \cdot \hat{\mathbf{a}},
\end{aligned}
$$

where $\mathbf{v}_{1} \cdot \mathbf{v}_{2}^{*}=0$ and $\hat{\mathbf{a}}=\left(\hat{a}_{1}, \hat{a}_{2}, \ldots, \hat{a}_{N}\right)$. From the full density matrix $\rho$ of the whole system, we form the output two-mode density matrix $\rho_{\text {out }}$ :

$$
\left\langle n m\left|\rho_{\text {out }}\right| n^{\prime} m^{\prime}\right\rangle=C \frac{\left\langle\operatorname{vac}\left|\hat{A}_{1}^{n} \hat{A}_{2}^{m} \rho \hat{A}_{2}^{\dagger m^{\prime}} \hat{A}_{1}^{\dagger n^{\prime}}\right| \mathrm{vac}\right\rangle}{\sqrt{n ! n^{\prime} ! m ! m^{\prime} !}},
$$

where $C$ is a normalization constant imposing $\operatorname{Tr}\left[\rho_{\text {out }}\right]=1$. For simplicity, here we consider that the reservoir is operating in the strongly interacting regime where the field $\hat{a}_{i}$ is effectively fermionized [27].

In Figs. 5(a)-5(c), we show the distribution of negativity for the prepared states for different reservoir sizes. We find that as the number of reservoir lattice sites $N$ increases, the output quantum state shows stronger entanglement with larger negativity $\operatorname{Ne}\left(\rho_{\text {out }}\right)$. Even for a given $N$, the negativity reaches up to its maximum value $\operatorname{Ne}\left(\rho_{\text {out }}\right)=N / 2$ with a significant probability [see Figs. 5(a)-5(c)]. Note that the maximum negativity $\operatorname{Ne}\left(\rho_{\text {out }}\right)=\left(D_{A}-1\right) / 2$, where $D_{A}=N+1$ is the effective Hilbert space dimension of the individual output mode. With the increasing reservoir size $N$, we obtain quantum states with stronger entanglement [larger $\left.\mathrm{Ne}\left(\rho_{\text {out }}\right)\right]$. However, the QRSP platform can also be used for preparing quantum states with a fixed negativity and other states (see SM [22]).

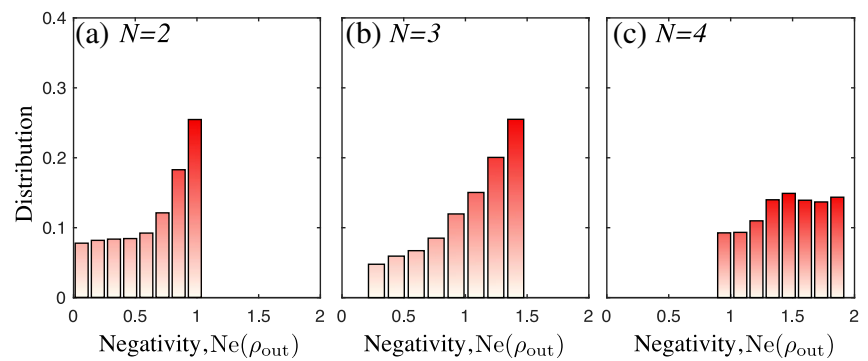

FIG. 5. Distribution of negativity of the output quantum states. We show the distributions of the negativity of quantum states $\mathrm{Ne}\left(\rho_{\text {out }}\right)$ prepared with different reservoir realizations and sizes $N=2$ (a), 3 (b), and 4 (c). The negativity of the prepared states increases with the increasing reservoir size. Here, we consider the parameters $\tau=1.2 \times 10^{-2} \hbar / \gamma, \tilde{J} / \gamma=10^{3}, E_{i}=0$ (for all sites), and $P_{i} / \gamma$ is taken uniformly distributed in $[-2.2,2.2] \times 10^{3}$. 
Discussion.-While preparing the considered states, we find that more complex quantum reservoirs are required for preparing the Schrödinger's cat states than those of the other two considered states. For instance, considering uniform on-site energies $E_{i}=0$ is enough to prepare the single-photon and entangled states; however, for preparing the cat states we required random on-site energies for achieving low preparation errors $\tilde{F}$. While we have considered small amplitudes for the cat states, this is only due to our limited (classical) resources for numerically simulating the quantum system. In principle, larger quantum reservoirs could be physically constructed and it is implied that in addition to increased performance they could access larger quantum states.

For physical implementation of our scheme, exciton polaritons in planar microcavities have shown to form interconnected networks [28-31] and also to reach the quantum nonlinear regime [23,32]. It can also be implemented in other systems, like arrays of quantum dots, trapped ions and atoms, nonlinear optical cavities, and networks of superconducting qubits where classical optical sources can interact with quantum nonlinear systems to generate quantized output fields.

The optimization procedure can either be performed numerically, simulating experimental results and providing target parameters for a physical system, or by using an experimentally measured cost function within the NelderMead procedure. We consider the effect of experimental errors in the latter process in the SM [22].

Although machine learning methods have been considered for optimizing universal quantum logic gate sets $[33,34]$ to prepare quantum states, with nonclassical input initial states [35], number resolved measurements [36], and conditional measurements [37], we stress that here we do not use such resources. Rather, we operate with only an irregular network of driven-dissipative Kerr nonlinear nodes, with classical coherent laser input.

Conclusion.-We have presented a quantum reservoir state preparation platform and demonstrated how it prepares various intriguing quantum states. This platform takes advantage of the large Hilbert space of a complex quantum reservoir. Taking weakly nonlinear bosonic lattices as the reservoir allows the enhancement of the unconventional blockade giving rise to single photons, while in strongly nonlinear lattices more exotic quantum superpositions and entangled states become available. Even considering disordered systems, site-to-site variations in local properties are not only overcome by the developed reservoir neural network framework but become a useful resource for quantum state preparation.

S. G. and T.C.H.L. were supported by the Ministry of Education (Singapore), Grant No. MOE2017-T2-1-001. S. G. thanks Debarati Neogy for help in preparing the figures. *sanjib.ghosh@ntu.edu.sg

†timothyliew@ntu.edu.sg

[1] R. Bedington, J. M. Arrazola, and A. Ling, npj Quantum Inf. 3, 30 (2017).

[2] S. L. Braunstein and P. van Loock, Rev. Mod. Phys. 77, 513 (2005).

[3] A. Steane, Rep. Prog. Phys. 61, 117 (1998).

[4] S. K. Liao et al., Phys. Rev. Lett. 120, 030501 (2018).

[5] X. Gao and L.-M. Duan, Nat. Commun. 8, 662 (2017).

[6] G. Carleo and M. Troyer, Science 355, 602 (2017).

[7] A. Nagy and V. Savona, Phys. Rev. Lett. 122, 250501 (2019).

[8] F. Vicentini, A. Biella, N. Regnault, and C. Ciuti, Phys. Rev. Lett. 122, 250503 (2019).

[9] P. Rotondo, M. Marcuzzi, J. P. Garrahan, I. Lesanovsky, and M. Müller, J. Phys. A 51, 115301 (2018).

[10] G. Tanaka, T. Yamane, J. B. Héroux, R. Nakane, N. Kanazawa, S. Takeda, H. Numata, D. Nakano, and A. Hirose, Neural Netw. 115, 100 (2019).

[11] C. Du, F. Cai, M. A. Zidan, W. Ma, S. H. Lee, and W. D. Lu, Nat. Commun. 8, 2204 (2017).

[12] H. Nomura, T. Furuta, K. Tsujimoto, Y. Kuwabiraki, F. Peper, E. Tamura, S. Miwa, M. Goto, R. Nakatani, and Y. Suzuki, Jpn. J. Appl. Phys. 58, 070901 (2019).

[13] J. Vatin, D. Rontani, and M. Sciamanna, Opt. Express 27, 18579 (2019).

[14] H. Jaeger, GMD Rep. 159, 48 (2002).

[15] M. Lukoševičius, A Practical Guide to Applying Echo State Networks, in Neural Networks: Tricks of the Trade, 2nd ed., edited by G. Montavon, G. B. Orr, and K.-R. Müller (Springer, Berlin, 2012), pp. 659-686.

[16] K. Nakajima, K. Fujii, M. Negoro, K. Mitarai, and M. Kitagawa, Phys. Rev. Applied 11, 034021 (2019).

[17] S. Ghosh, A. Opala, M. Matuszewski, T. Paterek, and T. C. H. Liew, npj Quantum Inf. 5, 35 (2019).

[18] H. Flayac and V. Savona, Phys. Rev. A 88, 033836 (2013).

[19] T. C. H. Liew and V. Savona, Phys. Rev. Lett. 104, 183601 (2010).

[20] M. Bamba, A. Imamoğlu, I. Carusotto, and C. Ciuti, Phys. Rev. A 83, 021802(R) (2011).

[21] J. C. Lagarias, J. A. Reeds, M. H. Wright, and P. E. Wright, SIAM J. Optim. 9, 112 (1998).

[22] See Supplemental at http://link.aps.org/supplemental/10 .1103/PhysRevLett.123.260404, for details on the solution of the quantum master equation, representations of different quantum states, the training procedure (including choice of cost function), and a consideration of the effect of errors.

[23] A. Delteil, T. Fink, A. Schade, S. Höfling, C. Schneider, and A. İmamoğlu, Nat. Mater. 18, 219 (2019).

[24] H. J. Snijders, J. A. Frey, J. Norman, H. Flayac, V. Savona, A. C. Gossard, J. E. Bowers, M. P. van Exter, D. Bouwmeester, and W. Löffler, Phys. Rev. Lett. 121, 043601 (2018).

[25] C. Vaneph, A. Morvan, G. Aiello, M. Féchant, M. Aprili, J. Gabelli, and J. Estève, Phys. Rev. Lett. 121, 043602 (2018).

[26] H. Flayac and V. Savona, Phys. Rev. A 96, 053810 (2017).

[27] I. Carusotto, D. Gerace, H. E. Tureci, S. De Liberato, C. Ciuti, and A. Imamoğlu, Phys. Rev. Lett. 103, 033601 (2009). 
[28] J. D. Töpfer, H. Sigurdsson, L. Pickup, and P. G. Lagoudakis, arXiv:1905.09092.

[29] H. Ohadi, Y. del Valle-Inclan Redondo, A. J. Ramsay, Z. Hatzopoulos, T. C. H. Liew, P. R. Eastham, P. G. Savvidis, and J. J. Baumberg, Phys. Rev. B 97, 195109 (2018).

[30] S. Klembt, T. H. Harder, O. A. Egorov, K. Winkler, R. Ge, M. A. Bandres, M. Emmerling, L. Worschech, T. C. H. Liew, M. Segev, C. Schneider, and S. Höfling, Nature (London) 562, 552 (2018).

[31] M. Milićević, T. Ozawa, G. Montambaux, I. Carusotto, E. Galopin, A. Lemaître, L. Le Gratiet, I. Sagnes, J. Bloch, and A. Amo, Phys. Rev. Lett. 118, 107403 (2017).

[32] Á. Cuevas, J. C. López Carreño, B. Silva, M. De Giorgi, D. G. Suárez-Forero, C. Sánchez Muñoz, A. Fieramosca, F. Cardano, L. Marrucci, V. Tasco, G. Biasiol, E. del Valle,
L. Dominici, D. Ballarini, G. Gigli, P. Mataloni, F. P. Laussy, F. Sciarrino, and D. Sanvitto, Sci. Adv. 4, eaao6814 (2018).

[33] J. M. Arrazola, T. R. Bromley, J. Izaac, C. R. Myers, K. Brádler, and N. Killoran, Quantum Sci. Technol. 4, 024004 (2019).

[34] N. Killoran, T. R. Bromley, J. M. Arrazola, M. Schuld, N. Quesada, and S. Lloyd, Phys. Rev. Research 1, 033063 (2019).

[35] K. K. Sabapathy, H. Qi, J. Izaac, and C. Weedbrook, Phys. Rev. A 100, 012326 (2019).

[36] L. O'Driscoll, R. Nichols, and P. A. Knott, Quantum Mach. Intell. 1, 5 (2019).

[37] R. Nichols, L. Mineh, J. R. Jiménez, J. Matthews, and P. Knott, Quantum Sci. Technol. 4, 045012 (2019). 